\title{
Schwannome de la langue
}

\author{
Keiichi Ohta DMD, Hitoshi Yoshimura DMD PhD
}

Citation : CMAJ 2021 January 18;193:E98. doi : 10.1503/cmaj.201039-f

Voir la version anglaise de l'article ici : www.cmaj.ca/lookup/doi/10.1503/cmaj.201039

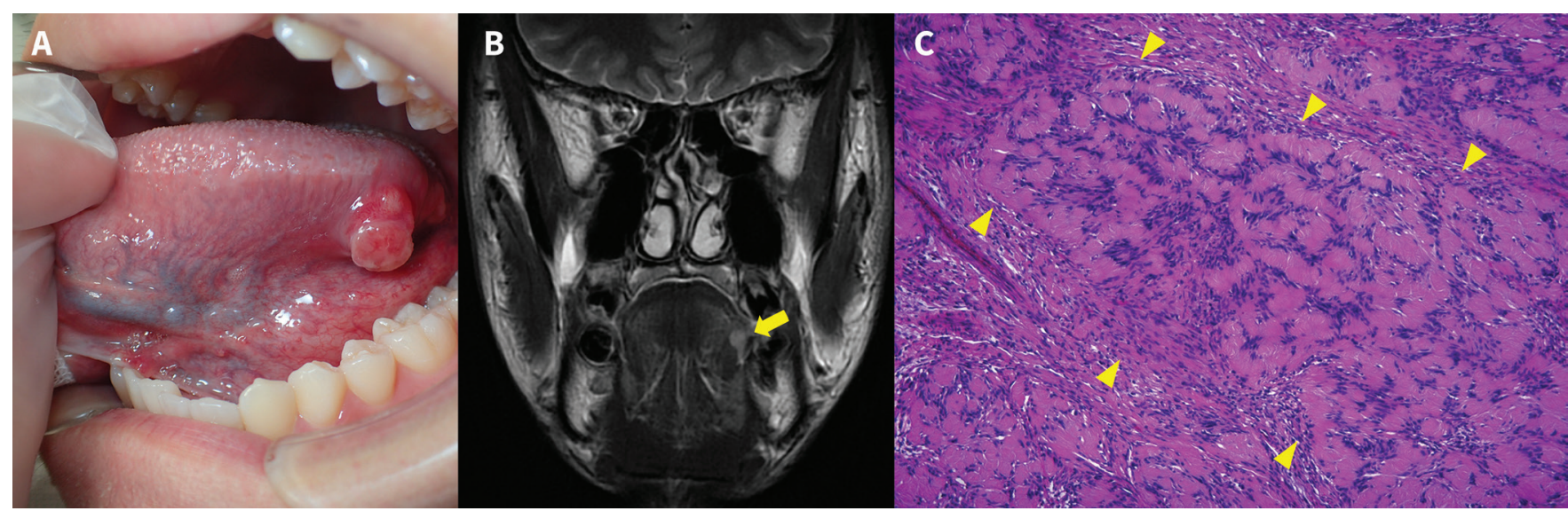

Figure 1 : A) Photographie d'une masse élastique bien circonscrite de $14 \mathrm{~mm} \times 9 \mathrm{~mm}$ sur le côté gauche de la langue d'un jeune homme de 17 ans atteint d'un schwannome. B) Imagerie par résonance magnétique montrant une lésion bien définie hyperintense en pondération T2 (flèche). C) Examen histopathologique avec coloration à l'hématoxyline et à l'éosine montrant une prolifération de cellules fusiformes (flèches) formant des palissades (Antoni A) [image grossie 100 fois].

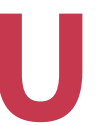
n jeune homme de 17 ans en santé est venu consulter pour une glossalgie latérale gauche présente depuis 2 semaines. À l'examen buccal, un nodule élastique de $14 \mathrm{~mm} \times 9 \mathrm{~mm}$, bien circonscrit et sensible à la palpation, était visible sur le côté gauche de la langue (figure $1 \mathrm{~A}$ ). Aucune lymphadénopathie cervicale n'a été constatée à l'examen physique. Un examen d'imagerie par résonance magnétique (IRM) a mis en évidence une lésion linguale gauche bien définie, iso-intense par rapport au muscle en pondération $T_{1}$ et hyperintense en pondération $T_{2}$ (figure 1B). L'examen histopathologique d'un échantillon prélevé par biopsie simple a montré des zones caractérisées par la prolifération de cellules fusiformes formant des palissades (figure 1C). Les analyses immunochimiques ont révélé la présence des marqueurs S100 et Sox10. La masse a été complètement excisée, et aucun signe de récidive n'était présent 10 mois plus tard.

Un schwannome est une tumeur bénigne des cellules de Schwann, qui forment la gaine des nerfs ${ }^{1}$. De $25 \%$ à $45 \%$ des schwannomes touchent la tête ou le cou ${ }^{1}$. Ils surviennent rarement dans la bouche, mais lorsque c'est le cas, ils se développent généralement sur la langue ${ }^{1}$. Les schwannomes de la langue s'observent habituellement chez les personnes de 20 à 40 ans, sans différence entre les sexes ${ }^{1}$. Les lésions se développent lentement, souvent sous forme de nodules sous-muqueux asymptomatiques, mais les grosses tumeurs sur la partie postérieure de la langue peuvent être à l'origine de douleurs, de dysphagie et de dysphonie ${ }^{1}$. Il est difficile de déterminer quels nerfs sont touchés par un schwannome buccal, la tumeur se développant la plupart du temps sur de petits nerfs ${ }^{2}$. La tomodensitométrie montrera des masses homogènes denses et bien circonscrites, tandis que l'IRM permettra un examen plus poussé ${ }^{1}$. Il existe 2 types histologiques de schwannomes: Antoni A et Antoni B. Le type A est caractérisé par la prolifération de cellules fusiformes formant des palissades, et le type B, par des zones hypocellulaires où l'arrangement des cellules est désorganisé1-3. Les marqueurs immunohistochimiques S100 et Sox10 sont utiles pour diagnostiquer les tumeurs touchant les gaines des nerfs périphériques 4 . Ils permettent tous deux la différenciation des cellules de la crête neurale, mais Sox10 est un marqueur plus précis que S100. Le traitement habituel des schwannomes est l'excision complète. Les récidives et la transformation en tumeur maligne sont très rares ${ }^{1}$. Toutes les lésions de la langue devraient faire l'objet d'une biopsie ou d'une excision, même chez les jeunes personnes, en raison de la possibilité, bien que rare, de cancer. 


\section{Références}

1. Kavčič J, Božič M. Schwannoma of the tongue. BMJ Case Rep 2016;2016: bcr2016215799.

2. Shashikumar T, Tejaswini JS, Bellad S, et al. Ancient schwannoma: a rare intramural intra-lingual lesion. Indian J Otolaryngol Head Neck Surg 2019;71: 816-9.
3. Thompson LDR, Koh SS, Lau SK. Tongue schwannoma: a clinicopathologic study of 19 cases. Head Neck Pathol 2020;14:571-6.

4. Karamchandani JR, Nielsen TO, van de Rijn M, et al. Sox10 and S100 in the diagnosis of soft-tissue neoplasms. Appl Immunohistochem Mol Morphol 2012;20:445-50.
Intérêts concurrents : Aucun n'a été déclaré.

Cet article a été révisé par des pairs.

Les auteurs ont obtenu le consentement du patient.

Affiliations : Département de médecine dentaire et de chirurgie buccale, Unité de médecine sensorielle et locomotrice, Division de médecine, Faculté des sciences médicales, Université de Fukui, Fukui, Japon.
Propriété intellectuelle du contenu : Il s'agit d'un article en libre accès distribué conformément aux modalités de la licence Creative Commons Attribution (CC BY-NC-ND 4.0), qui permet l'utilisation, la diffusion et la reproduction dans tout médium à la condition que la publication originale soit adéquatement citée, que l'utilisation se fasse à des fins non commerciales (c.-à-d., recherche ou éducation) et qu'aucune modification ni adaptation n'y soit apportée. Voir : https:// creativecommons.org/licenses/by-nc-nd/4.0/deed.fr.

Correspondance : Keiichi Ohta, kohta@g.u-fukui.ac.jp 